\title{
Aortic Stiffness, Cerebrovascular Dysfunction, and Memory
}

\author{
Leroy L. Cooper ${ }^{\mathrm{a}, \mathrm{b}} \quad$ Gary F. Mitchella \\ ${ }^{a}$ Cardiovascular Engineering, Inc., Norwood, Mass., and ${ }^{\mathrm{b}}$ Lifespan Cardiovascular Institute, \\ W. Alpert Medical School of Brown University, Providence, R.I., USA
}

\section{Key Words}

Aortic stiffness $\cdot$ Cognitive function $\cdot$ Memory $\cdot$ Pulsatile hemodynamics

\begin{abstract}
Background: Aortic stiffness is associated with cardiovascular and cerebrovascular events and cognitive decline. This mini-review focuses on relations of aortic stiffness with microvascular dysfunction and discusses the contribution of abnormal pulsatile hemodynamics to cerebrovascular damage and cognitive decline. We also provide a rationale for considering aortic stiffness as a putative and important contributor to memory impairment in older individuals. Summary: Aging is associated with stiffening of the aorta but not the muscular arteries, which reduces wave reflection and increases the transmission of pulsatility into the periphery. Aortic stiffening thereby impairs a protective mechanism that shields the peripheral microcirculation from excessive pulsatility within downstream target organs. Beyond midlife, aortic stiffness increases rapidly and exposes the cerebral microcirculation to abnormal pulsatile mechanical forces that are associated with microvascular damage and remodeling in the brain. Aortic stiffening and high-flow pulsatility are associated with alterations in the microvasculature of the brain; however, a mechanistic link between aortic stiffness and memory has not been established. We showed that in a community-based sample of older individuals, cerebrovascular resistance and white matter hyperintensities - markers of cerebrovascular remodeling and damage - mediated the relation between higher aortic stiffness and lower performance on memory function tests. These data suggest that microvascular and white matter damage associated with excessive aortic stiffness contribute to impaired memory function with advancing age. Key Messages: Increasing evidence suggests that vascular etiologies - including aortic stiffness and microvascular damage - contribute to memory impairment and the pathogenesis of dementia, including Alzheimer's disease. Interventions that reduce aortic stiffness may delay memory decline among older individuals.
\end{abstract}


Cooper and Mitchell: Aortic Stiffness, Cerebrovascular Dysfunction, and Memory

\section{Introduction}

Age is the foremost common risk factor for dementia. As the population ages, cognitive impairment is becoming more prevalent, resulting in a growing social and economic burden [1]. Along with degenerative changes in the brain, aging is associated with alterations in the structure and function of blood vessels, including a dramatic increase in aortic stiffness. Increased aortic stiffness is associated with elevated risk of incident cardiovascular and cerebrovascular disease events, including stroke [2-6]. Cerebrovascular pathologies are independently associated with cognitive dysfunction among the elderly. Oftentimes, vascular dysfunction manifests together with other disease markers, such as accumulation of $\beta$-amyloid plaques and tau protein tangles common to Alzheimer's disease (AD) [7, 8]. However, our current understanding of underlying mechanisms that link vascular dysfunction to agerelated cognitive decline remains incomplete.

We recently examined the interrelations of aortic stiffness, microvascular dysfunction, and memory in a community-based sample of older individuals [9]. We found that markers of cerebrovascular remodeling and damage provide a putative mechanism whereby aortic stiffness may affect performance in memory function tests. Thus, small vessel disease (SVD) and white matter damage associated with excessive aortic stiffness contribute to impaired memory with age. Vascular risk factors that are prominently associated with various dementias, including AD, represent important independent and potentially modifiable targets for prevention and treatment $[1,10,11]$. The treatment options for aortic stiffness and their implications have been reviewed previously $[12,13]$. The purpose of this review is to focus on the relation of aortic stiffness with microvascular dysfunction, to discuss the integral role of pulsatile hemodynamics and cerebrovascular damage in the pathogenesis of mild cognitive impairment and dementia, and to provide a rationale for considering aortic stiffness as a putative and important contributor to memory impairment in older individuals.

\section{Aortic Stiffness, Impedance Matching, and Microvascular Dysfunction}

Many methods of assessing arterial stiffness exist. Pulse wave velocity (PWV) is directly related to arterial stiffness and is easily assessed as the speed of a pulse wave traveling between two selected sites. The reference standard measure for aortic stiffness is carotidfemoral PWV (CFPWV), which is the measurement of PWV between the carotid and femoral arteries. CFPWV directly represents the stiffness of the thoracic and abdominal aorta and can be assessed noninvasively in the clinic using arterial tonometry and relatively modest equipment and expertise. Tonometric CFPWV is a potent novel indicator of cardiovascular disease (CVD) risk $[4,14]$. Conversely, measures of muscular artery PWV, such as carotidradial (muscular artery) and femoral-posterior tibial artery (femorotibial) PWV, which are much higher than CFPWV (aortic PWV) in young, healthy adults, are not related to incident CVD $[4,15]$.

Fragmentation of elastic fibers contributes to the progressive stiffening of the proximal aorta with age. The loss of elastic fibers is irreversible, which suggests that clinical and public health strategies should focus on primary prevention earlier in life. Over a lifetime, the proximal thoracic aorta bears the brunt of the pulsatile strain from cardiac ejection; therefore, the proximal aorta is susceptible to earlier deterioration than peripheral arteries. Elastic fiber fragmentation is associated with deposition and progressive engagement of much stiffer collagen fibers within the wall, ultimately stiffening the wall of the aorta and resulting in higher central pulse pressure, characteristic impedance, and PWV and altered timing of the reflected wave. In a study of the Framingham Offspring and Third Generation cohorts, aortic 
stiffness, as assessed by CFPWV, was shown to increase modestly with age through midlife and dramatically thereafter [16]. An age-related increase in aortic stiffness is accompanied by only minor changes in the stiffness of muscular arteries $[16,17]$. Stiffening of the proximal aorta, relative to the muscular and resistance arteries, increases the transmission of pulsatility into the microcirculation, resulting in target organ damage $[17,18]$. Younger, healthy individuals, however, generally have a highly compliant aorta and relatively stiff muscular arteries, which creates a discontinuity of impedance to pulsatile flow at the transition between the aorta and first-generation arteries. This impedance mismatch reflects a portion of the pulsatile energy stored in the forward traveling wave, limiting its transmission into the peripheral vasculature. After midlife, the impedance of the aorta increases disproportionately to the muscular arteries, leading to impedance matching and a reduction in wave reflection. Thus, reduced proximal wave reflection associated with aging removes a protective mechanism that normally shields the peripheral microcirculation from excessive pulsatility.

In response to excessive pressure and flow pulsatility, the microvessels remodel and constrict. This compensatory mechanism protects the susceptible microcirculation from excessive pulsatile energy but also increases resistance and reduces blood flow [19,20]. In the Framingham study, elevated aortic stiffness and increased pressure pulsatility were related to higher forearm vascular resistance at rest and blunted microvascular reactivity in response to ischemic stress [21]. Additionally, individuals with stiff arteries have a labile blood pressure, which may exacerbate the effects of blunted microvascular reactivity [22]. Thus, the initial cumulative sequelae of elevated aortic stiffness manifest as subclinical microvascular damage, which is a consequence of blood pressure lability, blunted microvascular reactivity, impaired autoregulation of organ blood flow, microvascular ischemia, and tissue damage [23].

\section{Aortic Stiffness, Cerebrovascular Damage, and Memory}

Recently, aortic stiffness has been associated with an increased risk for damage to microvascular structure and function of various target organs, including the kidneys and brain [9, 24-28]. Several factors, including blood-brain barrier and endothelial dysfunction, hypertension, hypercholesterolemia, diabetes mellitus, and smoking, contribute to cerebral SVD in older adults; however, the mechanisms by which aortic stiffness leads to cognitive dysfunction are complex and still incompletely understood. The brain is characterized by high flow, which requires low microvascular impedance. Since precapillary resistance provides the other major protection from pulsatile microvascular damage, the low-impedance brain circulation is particularly vulnerable to elevated pressure and flow pulsatility [18]. Elevated aortic stiffness and impedance matching drive the transmission of excessive pulsatile pressure and flow energy into the brain and damage small vessels and tissue.

The vascular hypothesis of AD is supported by studies of risk factors, but few have examined specific vascular properties that may mediate associations between vascular risk factors, memory impairment, and AD risk. Our research team recently investigated relations between aortic stiffness, microvascular damage, and cognitive function in the communitybased Age, Gene/Environment Susceptibility - Reykjavik Study [9]. Multivariable models adjusted for vascular and cognitive risk factors showed that a higher CFPWV was related to lower memory scores but was not related to processing speed or executive function. Previous research suggests that vascular injury and ischemia of the brain manifest preferentially as a decline of executive function and processing speed rather than memory [28-32]. In our study, however, we found the strongest relations between aortic stiffness (CFPWV) and memory. Perhaps, in our cohort with a very high prevalence of hypertension and aortic stiffness, along 
with their advanced age, the variability in vascular and cognitive measures is already reduced to a level that begins to obscure relations between stiffness and executive function or processing speed. Additionally, the loss of fiber tracts due to ischemic cerebrovascular injury in deep white matter may affect the integrity of gray matter regions associated with memory [33-36]. Particularly, function of the hippocampus has been shown more recently to be negatively associated with higher intracranial arterial flow pulsatility in elderly individuals [37], which may contribute to microvascular damage and cognitive decline [24].

We investigated the putative mechanistic link between central hemodynamics and cognitive function to determine whether cerebral and cerebrovascular remodeling and damage mediate the relation between aortic stiffness and memory. Mediating variables are intermediary variables that may provide a link between an observed (direct) effect between a predictor and an outcome. Quantifying how the mediator affects the observed relation may shine light onto possible pathological mechanisms [38-40]. To investigate the putative biological mechanism underlying the association between aortic stiffness and memory functional tests, we performed statistical mediation analysis [39]. Our analysis demonstrated that mechanisms involving cerebrovascular resistance and white matter hyperintensities (WMH) accounted for almost half of the observed relation between elevated CFPWV and lower performance on memory tests, whereas the direct relation between CFPWV and memory was no longer significant. Our results suggest that among older individuals, pathways that include cerebral microvascular remodeling and parenchymal damage contribute to the associations between elevated aortic stiffness and memory. It is important to acknowledge that in the forgoing study, we employed both a cross-sectional design and statistical mediation, which limits our ability to establish temporal and causal relations between CFPWV, mediators, and memory. In addition, since several other mechanisms may lead to cerebral SVD among the elderly, aortic stiffness may be sufficient but not necessary for the development of subclinical disease and cognitive impairment.

\section{Perspectives}

Recent findings suggest that vascular factors are potential targets for the prevention of memory impairment and AD. Without adequate autoregulation or compensatory remodeling to dampen pulsatile energy, elevated aortic stiffness transmits excessive pressure and flow pulsatility into the aging brain. Once this pulsatile energy penetrates into the cerebral microcirculation, microvascular damage, remodeling, and rarefaction may contribute to downstream ischemia and parenchymal damage. We and others have posited that this detrimental phenomenon targets deep brain structures, such as white matter and basal ganglia of the thalamus. These tissues are more susceptible to excessive pulsatility as they are perfused by short, penetrating branches that arise directly from the basilar or internal carotid arteries via the circle of Willis. In contrast to the long, circuitous muscular arteries that comprise the pial arterial network, these deep penetrating vessels provide minimal damping of the pulsatile pressure and flow that enters from large conduit vessels. Thus, structures perfused by these short, deep penetrators are more vulnerable to pressure and flow pulsatility and, hence, to abnormalities in aortic stiffness and pulsatile central hemodynamic load. On the other hand, the cerebral cortex is somewhat more protected as it is perfused by the circuitous and highly branched pial arteries, which attenuate pressure and flow pulsatility prior to their arrival in downstream tissues of the cerebral cortex. Indeed, we have shown that elevated CFPWV was associated with an increased prevalence of subcortical infarcts but not cortical infarcts [9, 24]. We have not examined an association of CFPWV with hippocampal structure; however, a recent study by Tarumi et al. [41] showed lower hippocampal and deep white matter 
Fig. 1. Conceptual model based on the collective evidence showing how aortic stiffness contributes to cognitive impairment and incident neurological disease. $\mathrm{CVR}=$ Cerebrovascular resistance.

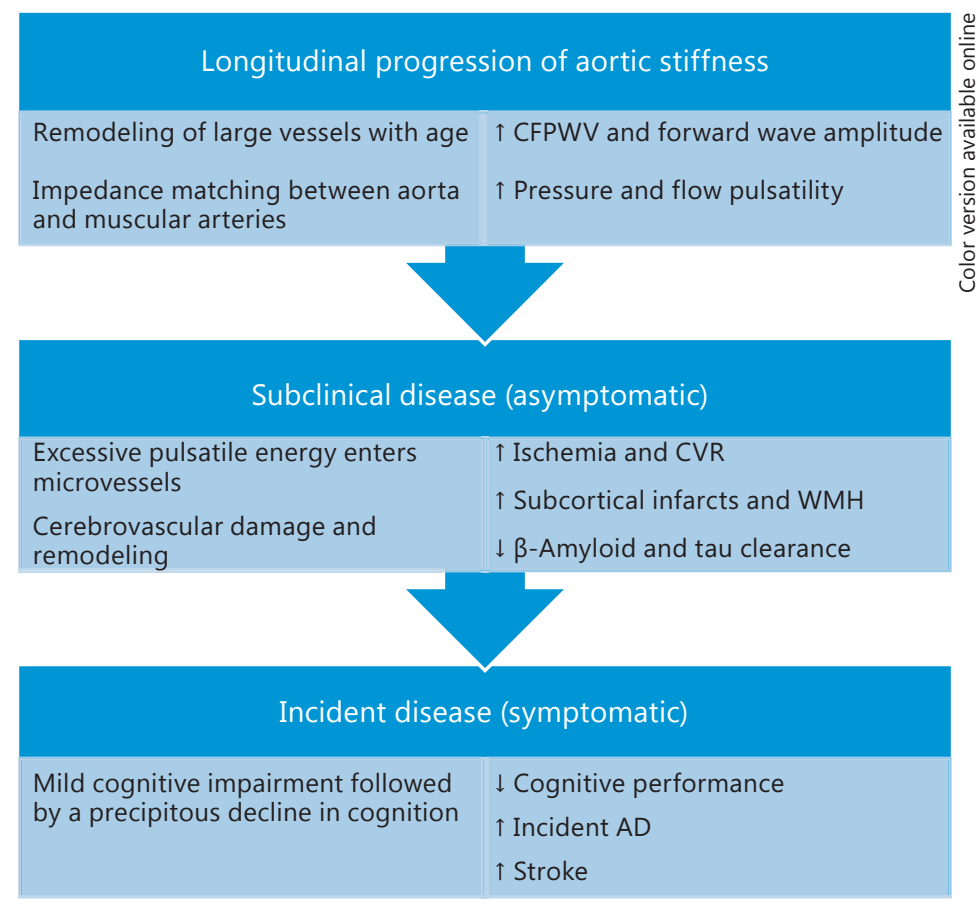

perfusion in individuals with elevated aortic stiffness. Since elderly individuals have blunted cerebral perfusion, an additional reduction of the cerebral blood flow due to cerebrovascular remodeling and impaired autoregulation may increase the risk for AD [42]. In addition to chronic hypoperfusion, pulsatile damage to small vessels in the brain may reduce the clearance of amyloid and tau proteins from the brain, possibly contributing to cerebral amyloid angiopathy and other protein clearance disorders associated with Alzheimer-type dementias [43]. Among middle-aged men, Shah et al. [44] showed that the $\beta$-amyloid-related risk for AD was higher with elevated diastolic blood pressure. In a study of elderly individuals without dementia, higher arterial stiffness and blood pressure were associated with higher $\beta$-amyloid deposition and more severe subclinical SVD [45]. Subsequently, Hughes et al. [46] showed that elevated arterial stiffness was related to progressive $\beta$-amyloid deposition in the brain of nondemented elderly adults over 2 years. In addition, Bangen et al. [47] revealed that the most common forms of cerebrovascular remodeling among patients with $\mathrm{AD}$ were atherosclerosis at the circle of Willis and arteriosclerosis. These data emphasize that remodeling of the aorta, elevated central hemodynamic load, and the subsequent damage and dysfunction of intracranial vessels convey a cumulative effect on brain structure and function that contribute to dementias, including $\mathrm{AD}$, with advancing age.

Figure 1 depicts a conceptual model showing how aortic stiffness may give rise to cognitive impairment and incident neurological disease. Structural evidence of subclinical SVD may be assessed with brain magnetic resonance imaging (MRI) and manifests as reduced brain volume and an increased burden of cerebral infarcts, WMH, lacunar infarcts, enlargement of the perivascular space, and compromised flow through the basilar and internal carotid arteries $[1,48]$. In addition, functional deficits associated with subclinical neurologic disease can be assessed by neuropsychological testing, which may reveal memory impairment - a precursor of dementia, including AD [7, 8, 49]. However, a crucial translational issue exists as to our ability to detect early-stage, subclinical cerebral SVD relatively quickly and at a reasonable cost in the clinic. Aortic stiffness, indicated by CFPWV, correlates with MRI 
markers of subclinical microvascular dysfunction and can be measured easily in the clinic noninvasively. However, the utility of CFPWV alone for the detection of early-stage cerebral SVD is likely insufficient. We posit that CFPWV, along with other vascular and nonvascular factors, could be a component of a comprehensive risk assessment tool that would be sensitive enough to stratify patients on increasing cerebral SVD risk. Additional preventative strategies for individuals at higher risk may include evaluation and monitoring via MRI and cognitive examinations, which are more expensive and time consuming. In light of an ever-growing chorus of physiological and epidemiological data linking aortic stiffness to cognitive impairment, screening for cognitive decline among middle-aged individuals who have elevated CVD risk along with prevalent elevated aortic stiffness may be advisable [50].

Since subtler alterations in vascular function may occur before midlife, when the transition to accelerated aortic stiffening occurs [16], additional studies using a broad range of participants are needed in order to establish whether associations between aortic stiffness and memory are modified by age. Further studies to characterize the neurocognitive consequences of higher aortic stiffness and secondary microvascular brain damage are also warranted. Currently, no thorough examination of the effects of increased aortic stiffness and excessive pressure and flow pulsatility on global and regional brain blood flow and cognitive function exists. Recently, aortic stiffness was shown to be independently associated with orthostatic hypotension in a sample of older participants of the Rotterdam Study [5]. In light of the foregoing relations between aortic stiffness and impaired microvascular reactivity, individuals with a stiffened aorta also may be susceptible to adverse consequences of orthostatic hypotension. However, relations of orthostatic changes in blood pressure with brain structure and function have not been studied thoroughly. Identifying additional vascular factors that contribute to early alterations to brain integrity may lead to preventable measures before the manifestation of symptomatic cognitive impairment. Since the role of aortic stiffness in the putative associations between orthostatic changes in blood pressure and brain structure and memory function remains unknown, assessment of the interrelations of orthostatic blood pressure change, aortic stiffness, and brain structure and function is merited.

\section{Conclusion}

Vascular factors, including elevated aortic stiffness and microvascular dysfunction, contribute to memory impairment. The prevalence of vascular risk factors and subclinical disease is rising as the population ages, and unfortunately, these risk factors often remain undiagnosed or untreated as they are asymptomatic [51]. Fortunately, vascular factors are modifiable targets. Because of competing risks in older people, it is possible that only a modest reduction in vascular dysfunction and an associated delay in onset of cognitive impairment could markedly reduce the prevalence of dementia. Since current therapies to treat advanced neurocognitive impairment and neurodegeneration are relatively ineffective, interventions designed to modify vascular factors that focus on large-vessel and cerebrovascular function may represent a critical opportunity to prevent or delay memory impairment and the onset of AD.

\section{Acknowledgements}

Dr. Cooper is supported by the UNCF/Merck Science Initiative. 
Cooper and Mitchell: Aortic Stiffness, Cerebrovascular Dysfunction, and Memory

\section{Disclosure Statement}

Dr. Mitchell is owner of Cardiovascular Engineering, Inc., a company that develops and manufactures devices to measure vascular stiffness, serves as a consultant to and receives honoraria and research grants from Novartis, Merck, Servier, and Philips, and is funded by research grants HL094898, DK082447, HL107385, HL104184, and HL126136 from the National Institutes of Health.

\section{References}

1 Gorelick PB, Scuteri A, Black SE, Decarli C, Greenberg SM, Iadecola C, Launer LJ, Laurent S, Lopez OL, Nyenhuis D, Petersen RC, Schneider JA, Tzourio C, Arnett DK, Bennett DA, Chui HC, Higashida RT, Lindquist R, Nilsson PM, Roman GC, Sellke FW, Seshadri S; American Heart Association Stroke Council, Council on Epidemiology and Prevention, Council on Cardiovascular Nursing, Council on Cardiovascular Radiology and Intervention, and Council on Cardiovascular Surgery and Anesthesia: Vascular contributions to cognitive impairment and dementia: a statement for healthcare professionals from the American Heart Association/American Stroke Association. Stroke 2011;42:2672-2713.

-2 Laurent S, Boutouyrie P, Asmar R, Gautier I, Laloux B, Guize L, Ducimetiere P, Benetos A: Aortic stiffness is an independent predictor of all-cause and cardiovascular mortality in hypertensive patients. Hypertension 2001; 37:1236-1241.

-3 Laurent S, Katsahian S, Fassot C, Tropeano AI, Gautier I, Laloux B, Boutouyrie P: Aortic stiffness is an independent predictor of fatal stroke in essential hypertension. Stroke 2003;34:1203-1206.

-4 Mitchell GF, Hwang SJ, Vasan RS, Larson MG, Pencina MJ, Hamburg NM, Vita JA, Levy D, Benjamin EJ: Arterial stiffness and cardiovascular events: the Framingham Heart Study. Circulation 2010;121:505-511.

-5 Mattace-Raso FU, van der Cammen TJ, Hofman A, van Popele NM, Bos ML, Schalekamp MA, Asmar R, Reneman RS, Hoeks AP, Breteler MM, Witteman JC: Arterial stiffness and risk of coronary heart disease and stroke: the Rotterdam Study. Circulation 2006;113:657-663.

6 Sutton-Tyrrell K, Najjar SS, Boudreau RM, Venkitachalam L, Kupelian V, Simonsick EM, Havlik R, Lakatta EG, Spurgeon H, Kritchevsky S, Pahor M, Bauer D, Newman A; Health ABC Study: Elevated aortic pulse wave velocity, a marker of arterial stiffness, predicts cardiovascular events in well-functioning older adults. Circulation 2005;111:3384-3390.

7 Zlokovic BV: Neurovascular pathways to neurodegeneration in Alzheimer's disease and other disorders. Nat Rev Neurosci 2011;12:723-738.

8 Iadecola C: The pathobiology of vascular dementia. Neuron 2013;80:844-866.

-9 Cooper LL, Woodard T, Sigurdsson S, van Buchem MA, Torjesen AA, Inker LA, Aspelund T, Eiriksdottir G, Harris TB, Gudnason V, Launer LJ, Mitchell GF: Cerebrovascular damage mediates relations between aortic stiffness and memory. Hypertension 2016;67:176-182.

10 Gorelick PB: Risk factors for vascular dementia and Alzheimer disease. Stroke 2004;35:2620-2622.

-11 Snyder HM, Corriveau RA, Craft S, Faber JE, Greenberg SM, Knopman D, Lamb BT, Montine TJ, Nedergaard M, Schaffer CB, Schneider JA, Wellington C, Wilcock DM, Zipfel GJ, Zlokovic B, Bain LJ, Bosetti F, Galis ZS, Koroshetz W, Carrillo MC: Vascular contributions to cognitive impairment and dementia including Alzheimer's disease. Alzheimers Dement 2015;11:710-717.

$\$ 12$ Adji A, O’Rourke MF, Namasivayam M: Arterial stiffness, its assessment, prognostic value, and implications for treatment. Am J Hypertens 2011;24:5-17.

13 Cattan V, Kakou A, Louis H, Lacolley P: Pathophysiology, genetic, and therapy of arterial stiffness. Biomed Mater Eng 2006;16:S155-S161.

14 Ben-Shlomo Y, Spears M, Boustred C, May M, Anderson SG, Benjamin EJ, Boutouyrie P, Cameron J, Chen CH, Cruickshank JK, Hwang SJ, Lakatta EG, Laurent S, Maldonado J, Mitchell GF, Najjar SS, Newman AB, Ohishi M, Pannier B, Pereira T, Vasan RS, Shokawa T, Sutton-Tyrell K, Verbeke F, Wang KL, Webb DJ, Willum Hansen T, Zoungas S, McEniery CM, Cockcroft JR, Wilkinson IB: Aortic pulse wave velocity improves cardiovascular event prediction: an individual participant meta-analysis of prospective observational data from 17,635 subjects. J Am Coll Cardiol 2014;63:636-646.

15 Pannier B, Guerin AP, Marchais SJ, Safar ME, London GM: Stiffness of capacitive and conduit arteries: prognostic significance for end-stage renal disease patients. Hypertension 2005;45:592-596.

16 Mitchell GF, Wang N, Palmisano JN, Larson MG, Hamburg NM, Vita JA, Levy D, Benjamin EJ, Vasan RS: Hemodynamic correlates of blood pressure across the adult age spectrum: noninvasive evaluation in the Framingham Heart Study. Circulation 2010;122:1379-1386.

17 Mitchell GF, Parise H, Benjamin EJ, Larson MG, Keyes MJ, Vita JA, Vasan RS, Levy D: Changes in arterial stiffness and wave reflection with advancing age in healthy men and women: the Framingham Heart Study. Hypertension 2004;43:1239-1245.

18 Mitchell GF: Effects of central arterial aging on the structure and function of the peripheral vasculature: implications for end-organ damage. J Appl Physiol 2008;105:1652-1660. 
19 Williamson GA, Loutzenhiser R, Wang X, Griffin K, Bidani AK: Systolic and mean blood pressures and afferent arteriolar myogenic response dynamics: a modeling approach. Am J Physiol Regul Integr Comp Physiol 2008; 295:R1502-R1511.

20 Loutzenhiser R, Bidani A, Chilton L: Renal myogenic response: kinetic attributes and physiological role. Circ Res 2002;90:1316-1324.

-21 Mitchell GF, Vita JA, Larson MG, Parise H, Keyes MJ, Warner E, Vasan RS, Levy D, Benjamin EJ: Cross-sectional relations of peripheral microvascular function, cardiovascular disease risk factors, and aortic stiffness: the Framingham Heart Study. Circulation 2005;112:3722-3728.

22 Schillaci G, Bilo G, Pucci G, Laurent S, Macquin-Mavier I, Boutouyrie P, Battista F, Settimi L, Desamericq G, Dolbeau G, Faini A, Salvi P, Mannarino E, Parati G: Relationship between short-term blood pressure variability and large-artery stiffness in human hypertension: findings from 2 large databases. Hypertension 2012;60: 369-377.

23 Mitchell GF: Cerebral small vessel disease: role of aortic stiffness and pulsatile hemodynamics. J Hypertens 2015;33:2025-2028.

-24 Mitchell GF, van Buchem MA, Sigurdsson S, Gotal JD, Jonsdottir MK, Kjartansson O, Garcia M, Aspelund T, Harris TB, Gudnason V, Launer LJ: Arterial stiffness, pressure and flow pulsatility and brain structure and function: the Age, Gene/Environment Susceptibility - Reykjavik Study. Brain 2011;134:3398-3407.

25 Woodard T, Sigurdsson S, Gotal JD, Torjesen AA, Inker LA, Aspelund T, Eiriksdottir G, Gudnason V, Harris TB, Launer LJ, Levey AS, Mitchell GF: Mediation analysis of aortic stiffness and renal microvascular function. J Am Soc Nephrol 2015;26:1181-1187.

26 Pase MP, Himali JJ, Mitchell GF, Beiser A, Maillard P, Tsao C, Larson MG, DeCarli C, Vasan RS, Seshadri S: Association of aortic stiffness with cognition and brain aging in young and middle-aged adults: the Framingham Third Generation Cohort Study. Hypertension 2016;67:513-519.

-27 Maillard P, Mitchell GF, Himali JJ, Beiser A, Tsao CW, Pase MP, Satizabal CL, Vasan RS, Seshadri S, DeCarli C: Effects of arterial stiffness on brain integrity in young adults from the Framingham Heart Study. Stroke 2016; 47:1030-1036.

28 Tsao CW, Himali JJ, Beiser AS, Larson MG, DeCarli C, Vasan RS, Mitchell GF, Seshadri S: Association of arterial stiffness with progression of subclinical brain and cognitive disease. Neurology 2016;86:619-626.

-29 Prins ND, van Dijk EJ, den Heijer T, Vermeer SE, Jolles J, Koudstaal PJ, Hofman A, Breteler MM: Cerebral smallvessel disease and decline in information processing speed, executive function and memory. Brain 2005; 128: 2034-2041.

-30 Jokinen H, Kalska H, Ylikoski R, Madureira S, Verdelho A, van der Flier WM, Scheltens P, Barkhof F, Visser MC, Fazekas F, Schmidt R, O’Brien J, Waldemar G, Wallin A, Chabriat H, Pantoni L, Inzitari D, Erkinjuntti T; LADIS Group: Longitudinal cognitive decline in subcortical ischemic vascular disease - the LADIS Study. Cerebrovasc Dis 2009;27:384-391.

-31 Poels MM, van Oijen M, Mattace-Raso FU, Hofman A, Koudstaal PJ, Witteman JC, Breteler MM: Arterial stiffness, cognitive decline, and risk of dementia: the Rotterdam Study. Stroke 2007;38:888-892.

32 Watson NL, Sutton-Tyrrell K, Rosano C, Boudreau RM, Hardy SE, Simonsick EM, Najjar SS, Launer LJ, Yaffe K, Atkinson HH, Satterfield S, Newman AB: Arterial stiffness and cognitive decline in well-functioning older adults. J Gerontol A Biol Sci Med Sci 2011;66:1336-1342.

-33 Grool AM, van der Graaf Y, Mali WP, Geerlings MI; SMART Study Group: Location of cerebrovascular and degenerative changes, depressive symptoms and cognitive functioning in later life: the SMART-Medea Study. J Neurol Neurosurg Psychiatry 2011;82:1093-1100.

-34 Schmidt R, Ropele S, Enzinger C, Petrovic K, Smith S, Schmidt H, Matthews PM, Fazekas F: White matter lesion progression, brain atrophy, and cognitive decline: the Austrian Stroke Prevention Study. Ann Neurol 2005;58: 610-616.

-35 Kloppenborg RP, Nederkoorn PJ, Geerlings MI, van den Berg E: Presence and progression of white matter hyperintensities and cognition: a meta-analysis. Neurology 2014;82:2127-2138.

-36 Jokinen H, Gouw AA, Madureira S, Ylikoski R, van Straaten EC, van der Flier WM, Barkhof F, Scheltens P, Fazekas F, Schmidt R, Verdelho A, Ferro JM, Pantoni L, Inzitari D, Erkinjuntti T; LADIS Group: Incident lacunes influence cognitive decline: the LADIS Study. Neurology 2011;76:1872-1878.

37 Wahlin A, Ambarki K, Birgander R, Malm J, Eklund A: Intracranial pulsatility is associated with regional brain volume in elderly individuals. Neurobiol Aging 2014;35:365-372.

-38 Mackinnon DP, Fairchild AJ: Current directions in mediation analysis. Curr Dir Psychol Sci 2009;18:16.

39 Hayes AF: An Introduction to Mediation, Moderation, and Conditional Process Analysis: A Regression-Based Approach. New York, Guilford Press, 2013.

40 Valeri L, VanderWeele TJ: SAS macro for causal mediation analysis with survival data. Epidemiology 2015; 26:e23-e24.

41 Tarumi T, Shah F, Tanaka H, Haley AP: Association between central elastic artery stiffness and cerebral perfusion in deep subcortical gray and white matter. Am J Hypertens 2011;24:1108-1113.

-42 Qiu CX, von Strauss E, Fastbom J, Winblad B, Fratiglioni L: Low blood pressure and risk of dementia in the Kungsholmen project - a 6-year follow-up study. Arch Neurol 2003;60:223-228.

-43 Carare RO, Hawkes CA, Jeffrey M, Kalaria RN, Weller RO: Review: cerebral amyloid angiopathy, prion angiopathy, CADASIL and the spectrum of protein elimination failure angiopathies (PEFA) in neurodegenerative disease with a focus on therapy. Neuropathol Appl Neurobiol 2013;39:593-611. 
44 Shah NS, Vidal JS, Masaki K, Petrovitch H, Ross GW, Tilley C, DeMattos RB, Tracy RP, White LR, Launer LJ: Midlife blood pressure, plasma $\beta$-amyloid, and the risk for Alzheimer disease: the Honolulu Asia Aging Study. Hypertension 2012;59:780-786.

-45 Hughes TM, Kuller LH, Barinas-Mitchell EJM, Mackey RH, McDade EM, Klunk WE, Aizenstein HJ, Cohen AD, Snitz BE, Mathis CA, DeKosky ST, Lopez OL: Pulse wave velocity is associated with $\beta$-amyloid deposition in the brains of very elderly adults. Neurology 2013;81:1711-1718.

-46 Hughes TM, Kuller LH, Barinas-Mitchell EJM, McDade EM, Klunk WE, Cohen AD, Mathis CA, DeKosky ST, Price JC, Lopez OL: Arterial stiffness and $\beta$-amyloid progression in nondemented elderly adults. JAMA Neurol 2014; 71:562-568.

47 Bangen KJ, Nation DA, Delano-Wood L, Weissberger GH, Hansen LA, Galasko DR, Salmon DP, Bondi MW: Aggregate effects of vascular risk factors on cerebrovascular changes in autopsy-confirmed Alzheimer's disease. Alzheimers Dement 2014;11:394-403.

-48 Spilt A, Box FMA, van der Geest RJ, Reiber JHC, Kunz P, Kamper AM, Blauw GJ, van Buchem MA: Reproducibility of total cerebral blood flow measurements using phase contrast magnetic resonance imaging. J Magn Reson Imaging 2002;16:1-5.

49 Kalaria RN, Akinyemi R, Ihara M: Does vascular pathology contribute to Alzheimer changes? J Neurol Sci 2012; 322:141-147.

50 Scuteri A, Stone J, O'Rourke MF: The relationship between aortic stiffness, microvascular disease in the brain and cognitive decline: insights into the emerging epidemic of Alzheimer's disease; in Safar ME, O'Rourke MF, Frohlich ED (eds): Blood Pressure and Arterial Wall Mechanics in Cardiovascular Diseases. London, Springer, 2014, pp 307-320.

51 DeCarli C: Clinically asymptomatic vascular brain injury: a potent cause of cognitive impairment among older individuals. J Alzheimers Dis 2013;33(suppl 1):S417-S426. 